\title{
Disrupted ciliated epithelium shows slower ciliary beat frequency and increased dyskinesia
}

\author{
B. Thomas, A. Rutman and C. O'Callaghan
}

ABSTRACT: Ciliary function studies for the diagnosis of primary ciliary dyskinesia (PCD) are usually performed on nasal brush biopsy samples. It is not uncommon to find disrupted epithelial strips of tissue in these samples, and occasionally throughout a sample. The aim of the present study was to determine if cilia on disrupted ciliated epithelial edges beat with a normal pattern and frequency similar to that of cilia on undisrupted edges.

Nasal brush biopsy samples from 42 children in whom the diagnosis of PCD was excluded were assessed. The epithelial strips were categorised into five groups: intact undisrupted ciliated epithelial edge, ciliated epithelial edge with minor projections, ciliated epithelial edge with major projections, an isolated ciliated cell on an epithelial edge and single unattached ciliated cells. Ciliary beat frequency and beat pattern of 50 samples from each group were determined using high speed digital video microscopy.

The cilia on epithelial edges with varying degrees of disruption showed significantly reduced beat frequency and significantly increased dyskinesia compared with those on intact, undisrupted ciliated epithelial edges.

Ideally, the assessment of ciliary beat pattern and frequency for PCD diagnosis should only be performed on undisrupted ciliated edges.

KEYWORDS: Ciliary beat frequency, ciliary beat pattern, primary ciliary dyskinesia, respiratory epithelium

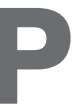

rimary ciliary dyskinesia (PCD) is an inherited disorder of ciliary motility with an incidence of $\sim 1$ in 15,000 in the Caucasian population [1, 2]. This genetically heterogeneous disorder may be caused by a variety of ciliary ultrastructural defects that reflect the complex array of structural proteins that form the normal ciliary axoneme [2,3]. The resulting abnormalities of ciliary motility cause impaired mucociliary clearance that accounts for the clinical phenotype, dominated by chronic symptoms involving the nose, middle ear, sinuses and the chest $[2,4]$. In conjunction with the history and clinical findings, the diagnosis of PCD is based on the assessment of ciliary beat frequency $(\mathrm{CBF})$, beat pattern and ultrastructure. Also, CBF and beat pattern are commonly used outcome measures in studies that assess the effect of pathogenic organisms or pharmacological agents on the ciliated respiratory epithelium. The recent advent of high speed digital video microscopy allows us to precisely assess the ciliary beat pattern, in addition to the measurement of $\mathrm{CBF}$ [5] and its use is being adopted internationally for the diagnostic testing of PCD [6].

Ciliary function studies for the diagnosis of PCD are usually performed on nasal brush biopsy samples. The technique is simple and in most cases, a good quality sample with a number of intact uniform ciliated epithelial edges is obtained. However, it is not uncommon to find disrupted edges with cellular projections and isolated ciliated cells in the sample obtained. Sometimes, disruption of ciliated edges may occur throughout an entire sample. The aim of the present study was to determine if cilia on disrupted ciliated epithelial edges beat with a normal beat pattern and frequency similar to that of cilia on undisrupted edges.

\section{AFFILIATIONS}

Dept of Infection, Immunity and Inflammation, University of Leicester Leicester Royal Infirmary, Leicester, UK.

CORRESPONDENCE C. O'Callaghan Dept of Infection, Immunity and Inflammation

University of Leicester PO Box 65 Leicester Royal Infirmary Leicester LE2 7LX UK E-mail: ajb64@le.ac.uk

Received: Oct 102008

Accepted after revision: March 112009 


\section{METHODS}

Nasal brush biopsy samples obtained from 42 children were studied. The sample quality was assessed and categorised into five groups (fig. 1): a normal edge (defined as an intact uniform ciliated epithelial strip $>50 \mu \mathrm{m}$ in length); a ciliated edge with minor projections (defined as an edge $>50 \mu \mathrm{m}$ in length, with cells projecting out of the epithelial edge line, but with no point of the apical cell membrane projecting above the tips of the cilia on the adjacent cells); a ciliated edge with major projections (defined as an edge $>50 \mu \mathrm{m}$ in length, with cells projecting out of the epithelial edge line, with at least one point of the apical cell membrane projecting above the tips of the cilia on the adjacent cells); an isolated ciliated cell (defined as the only ciliated cell on an epithelial edge $>50 \mu \mathrm{m}$ in length) and single cells (defined as ciliated cells that had no contact between themselves or any other cell type). We used this arbitrary categorisation in the assumption that the alignment of the cells and the degree of apposition between adjacent ciliated cells are likely to differ in these categories.
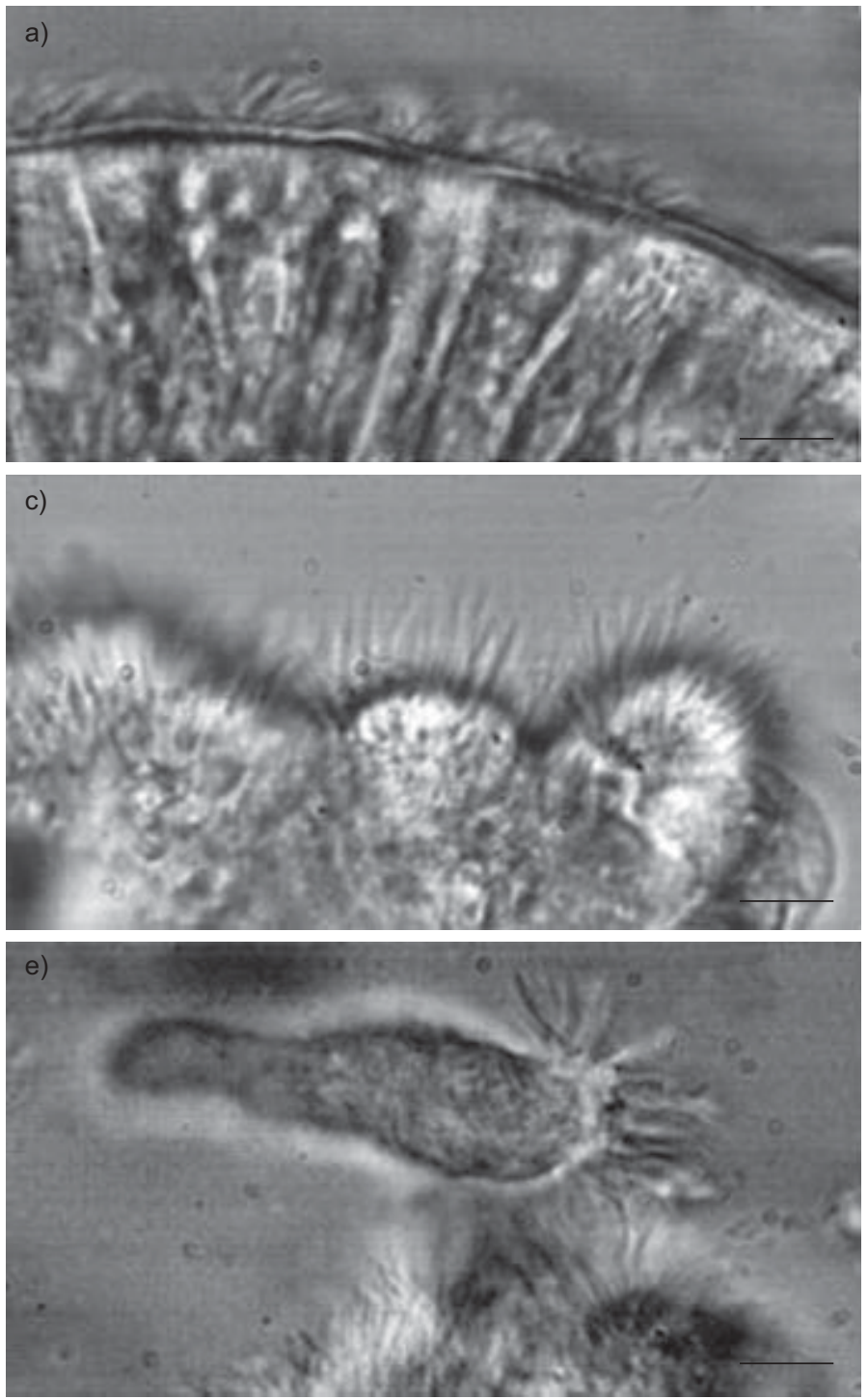

A sample that yielded a minimum of seven (up to a maximum of 10) normal ciliated epithelial edges is considered as adequate for ciliary function studies in our laboratory. The nasal brush biopsy sample from each of these 42 children contained at least seven normal epithelial edges for ciliary function studies. PCD was excluded in all 42 children by ciliary function studies and analysis of ultrastructure, using previously published methodology and normative data [5, 7]. The images recorded from these 42 children also included a variable number of disrupted epithelial edges that belong to the five different categories. The number of samples of each category varied between biopsy specimens and not all categories were always found in a single biopsy specimen. 50 samples that belong to each sample category were randomly selected and CBF was measured and beat pattern determined using a high speed digital video microscopy system.

Nasal brush biopsy samples were placed in Medium 199 (25 mM hydroxyethyl piperazine ethane sulphonic acid, Earles salt and L-glutamine (pH 7.3); Gibco, Leicester, UK) that
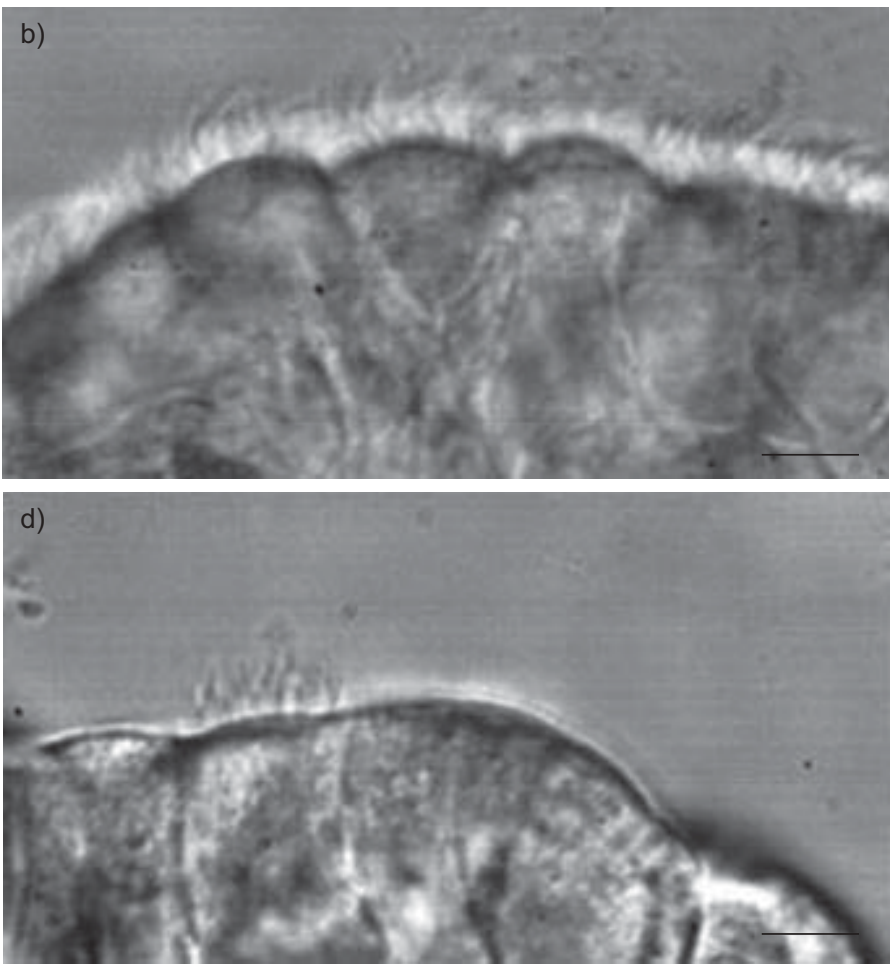

FIGURE 1. Representative images of different types of ciliated epithelial edges studied. a) Normal edge: intact, uniform ciliated epithelial strip $>50 \mu \mathrm{m}$ in length; b) minor projection: ciliated epithelial strip $>50 \mu \mathrm{m}$ in length, with cells projecting out of the epithelial edge line, but with no point of the apical cell membrane projecting above the tips of the cilia on the adjacent cells; c) major projection: defined as ciliated epithelial strip $>50 \mu \mathrm{m}$ in length, with cells projecting out of the epithelial edge line, with at least one point of the apical cell membrane projecting above the tips of the cilia on the adjacent cells; d) isolated ciliated cell: defined as the only ciliated cell on an epithelial strip $>50 \mu \mathrm{m}$ in length and e) single cell: defined as ciliated cells that had no contact between themselves or any other cell type. Scale bars $=5.5 \mu \mathrm{m}$. 
contained antibiotic solution (streptomycin $50 \mu \mathrm{g} \cdot \mathrm{mL}^{-1}$ and penicillin $50 \mu \mathrm{g} \cdot \mathrm{mL}^{-1}$ ) and suspended in a chamber created by the separation of a cover slip and a glass slide by two adjacent cover slips. The slide was placed on a heated stage $\left(37^{\circ} \mathrm{C}\right)$ of a Leitz Diaplan microscope mounted on an anti-vibration table (Wentworth Laboratories Ltd, Sandy, UK). Ciliated epithelium devoid of mucus was observed at $37^{\circ} \mathrm{C}$ using a $\times 100$ interference contrast lens. They were recorded using a digital high speed video camera (Kodak Ektapro Motion Analyser; Eastman Kodak, San Diego, CA, USA) at a frame rate of 400 frames $\cdot \mathrm{s}^{-1}$. Video sequences could be recorded and played back at reduced frame rates or frame by frame. CBF was determined directly from ciliated epithelial cells viewed in a sideways profile. Groups of beating cilia were identified and the number of frames required to complete 10 beat cycles was recorded. This was converted to $\mathrm{CBF}$ by a simple calculation $(\mathrm{CBF}=(400 /$ number frames for 10 beats $) \times 10)$ [5]. The experimental system allowed the ciliary beat pattern to be evaluated in three different planes: a sideways profile, beating directly toward the observer and from directly above $[5,7]$. The path taken by a cilium during the beat cycle was analysed frame by frame. This was characterised and compared with normal beat pattern [5] seen on digital high speed video analysis. Dyskinesia was defined as an abnormal beat pattern that included reduced beat amplitude, stiff beat pattern, failure to bend along the length of the ciliary shaft, flickering or a twitching motion and static cilia. The percentage of dyskinetic cilia in each sample was assessed and expressed as a dyskinesia score (0: normal beat pattern throughout the sample; 1: dyskinetic beat pattern in $\leqslant 25 \%$ of the cilia; 2 : dyskinetic beat pattern in $\leqslant 50 \%$ of the cilia; 3: dyskinetic beat pattern in $\leqslant 75 \%$ of the cilia; and 4 : dyskinetic beat pattern in all cilia). The videotapes were re-analysed by a second observer (B. Thomas) and blinded on a second occasion by the original observer (A. Rutman).

\section{Statistical analysis}

Statistical analysis was performed using GraphPad Prism 5 (GraphPad, San Diego, CA, USA). Nonparametric data were described as median (interquartile range (IQR)). ANOVA was performed using the Kruskal-Wallis test and the data groups were compared using the Mann-Whitney U-test. A p-value of $<0.05$ was taken as the threshold for statistical significance. Agreement between the two observers was excellent for CBF (interclass correlation 0.97 ) as well as dyskinesia index (interclass correlation 1.0). Repeatability (agreement within observer) was also excellent (interclass correlation was 0.998 for $\mathrm{CBF}$ and 1.0 for dyskinesia index).

\section{RESULTS}

The CBF (median (IQR)) of different types of ciliated epithelia was 13.4 (11.6-14.2) Hz for normal ciliated edge, 11.4 (8.913.9) $\mathrm{Hz}$ for ciliated edge with minor projections, 8.7 (7.311.3) $\mathrm{Hz}$ for ciliated edge with major projections, 9.2 (7.210.3) $\mathrm{Hz}$ for epithelial strip with an isolated ciliated cell and 6.5 (2.2-8.3) Hz for single unattached ciliated cells. The dyskinesia scores of different types of ciliated epithelia were 0 (0-1) for normal ciliated edge, 1 (0-2) for ciliated edge with minor projections, 2 (1-3) for ciliated edge with major projections, 2 (1.8-3) for epithelial strip with an isolated ciliated cell and 4 (34) for single unattached ciliated cells. The difference in ciliary beat frequency and dyskinesia scores between normal ciliated edge and all the other types of ciliated epithelia were statistically significant (fig. 2).

\section{DISCUSSION}

We have shown that the CBF and beat pattern assessed on cilia that are not part of an intact undisrupted strip of ciliated epithelium, differ significantly compared with that of an intact uniform ciliated epithelial edge. Disrupted ciliated epithelium showed a slower CBF and increased dyskinesia. We have observed similar abnormalities of $\mathrm{CBF}$ and beat pattern of cilia on disrupted epithelial edges obtained from air-liquid interface cultures (unpublished data).

In a previous study of functional analysis of cilia and ciliary ultrastructure in healthy children [5], a mean (95\% confidence
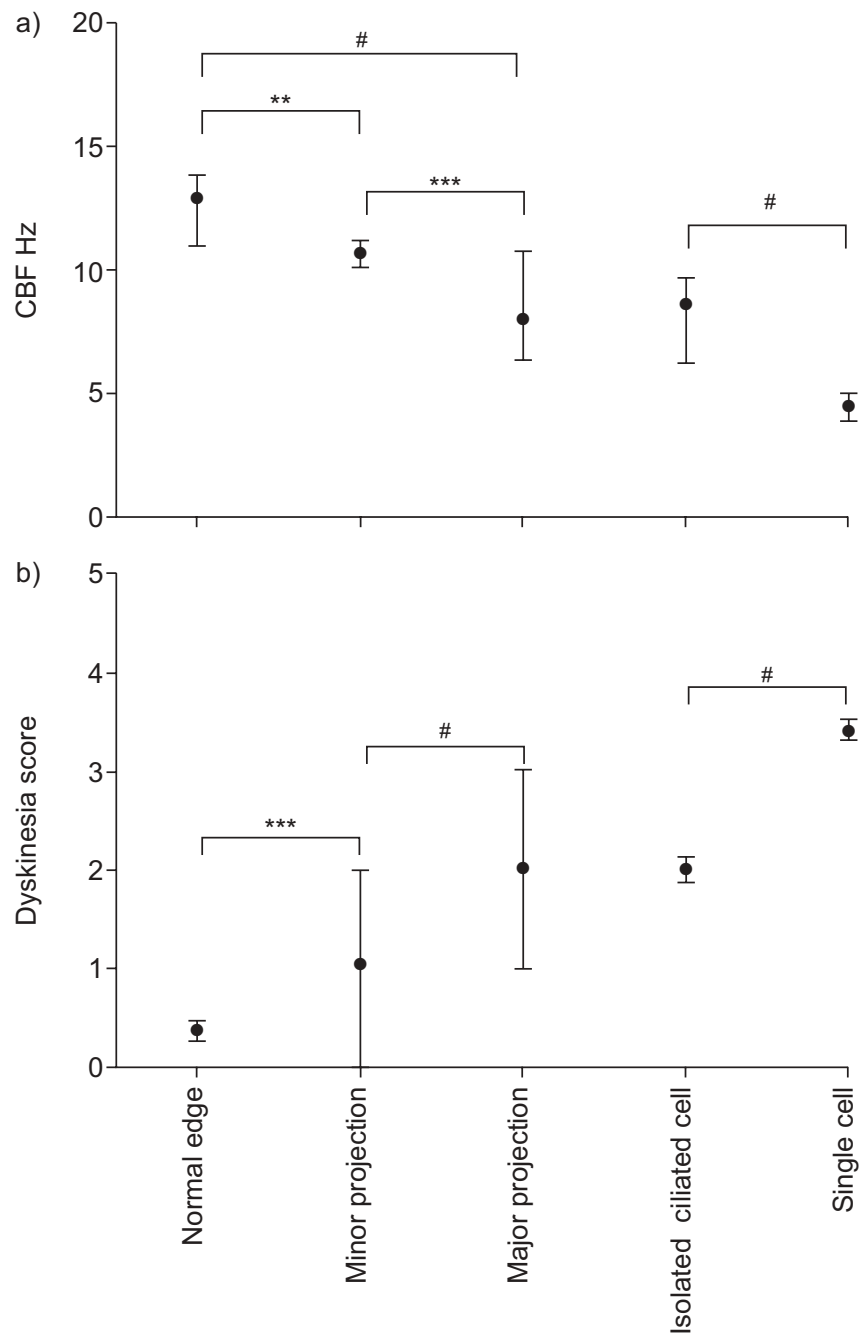

FIGURE 2. Ciliary beat frequency (CBF) and dyskinesia score for different types of ciliated epithelium. a) CBF and b) dyskinesia score (0: normal beat pattern throughout the epithelium; 1 : dyskinetic beat pattern in $\leqslant 25 \%$ of the cilia studied; 2 : dyskinetic beat pattern in $\leqslant 50 \%$ of the cilia studied; 3 : dyskinetic beat pattern in $\leqslant 75 \%$ of the cilia studied; and 4 : dyskinetic beat pattern in all cilia studied). Data are expressed as median (interquartile range). ${ }^{*}: p<0.0001 ; * *: p<0.01 ; * * *$ : $\mathrm{p}<0.001$. 
interval) $\mathrm{CBF}$ of $12.8(12.3-13.3) \mathrm{Hz}$ was reported and the proportion of cilia with ultrastructural defects was $<5 \%$. Also, we have previously shown that different ultrastructural defects seen in PCD results in predictable abnormalities of ciliary beat pattern [7]. However, the beat pattern of cilia on disrupted edges was highly variable and no characteristic beat pattern in relation to sample type was observed. This was the first study that objectively quantified the ciliary function (in terms of CBF and beat pattern) of ciliated epithelium with varying degrees of disruption. We did not aim to establish the normative data for CBF for disrupted ciliated epithelium.

It is well recognised that measurement of CBF may be affected by technical and methodological issues including environmental factors such as $\mathrm{pH}$ and temperature $[8,9]$. It appears from the published literature that another important, but less well appreciated factor is the effect of quality of the ciliated epithelia on ex vivo assessment of ciliary function. Though a number of authors have noted the variability of measured CBF between ciliated epithelia within a biopsy sample [10-14], this is the first study that has objectively quantified the ciliary function (in terms of CBF and beat pattern) of ciliated epithelium with varying degrees of disruption. Emerging evidence suggests that the ciliary beat is mechanosensitive and the normal metachronal ciliary beat wave is regulated by a complex interplay of intricate signalling pathways that involve physical and biochemical mechanisms [15]. Even local shear forces generated by the motion of adjacent cilia may have a role in this and our results support this notion.

Based on the current study, we suggest that ideally, only undisrupted strips of ciliated epithelium may be used for the assessment of CBF and beat pattern for the diagnostic testing for PCD. However, in many instances, this may not be achievable and in such circumstances, strips of ciliated epithelium with minor cellular projections may be used. We strongly discourage using ciliated epithelial strips with major cellular projections, isolated ciliated cells on epithelial strips or single ciliated cells for ciliary function studies. If $\mathrm{CBF}$ is measured and beat pattern assessed from cilia that are not part of an intact undisrupted strip of ciliated epithelium, this should be stated and the results should be viewed with caution. Our findings emphasise the need for inclusion of objective criteria regarding the quality of the ciliated epithelia studied, in the protocols used for ciliary function studies.

\section{STATEMENT OF INTEREST}

None declared.

\section{REFERENCES}

1 Sturgess JM, Thompson MW, Czegledy-Nagy E, et al. Genetic aspects of immotile cilia syndrome. Am J Med Genet 1986; 25: 149-160.

2 Bush A, Chodhari R, Collins N, et al. Primary ciliary dyskinesia: current state of the art. Arch Dis Child 2007; 92: 1136-1140.

3 Brody SL. Genetic regulation of cilia assembly and the relationship to human disease. Am J Respir Cell Mol Biol 2004; 30: 435-437.

4 Noone PG, Leigh MW, Sannuti A, et al. Primary ciliary dyskinesia: diagnostic and phenotypic features. Am J Respir Crit Care Med 2004; 169: 459-467.

5 Chilvers MA, Rutman A, O'Callaghan C. Functional analysis of cilia and ciliated epithelial ultrastructure in healthy children and young adults. Thorax 2003; 58: 333-338.

6 Ferkol T, Mitchison HM, O'Callaghan C, et al. Current issues in the basic mechanisms, pathophysiology, diagnosis and management of primary ciliary dyskinesia. In: Frey U, Gerritsen J. Respiratory Diseases in Infants and Children. Eur Respir Mon 2006; 37: 291-313.

7 Chilvers MA, Rutman A, O'Callaghan C. Ciliary beat pattern is associated with specific ultrastructural defects in primary ciliary dyskinesia. J Allergy Clin Immunol 2003; 112: 518-524.

8 Clary-Meinesz CF, Cosson J, Huitorel P, et al. Temperature effect on the ciliary beat frequency of human nasal and tracheal ciliated cells. Biol Cell 1992; 76: 335-338.

9 Salathe M. Regulation of mammalian ciliary beating. Annu Rev Physiol 2007; 69: 401-422.

10 Bryan WT, Bryan MP, Smith CA. Human ciliated epithelial cells in nasal secretions. Morphologic and histochemical aspects. Ann Otol Rhinol Laryngol 1964; 73: 474-487.

11 Lopez-Vidriero M. Objective criteria for measuring ciliary beat frequency in vitro. Am Rev Respir Dis 1982; 125: Suppl. 4, 244.

12 Roth Y, Ostfeld E. Ciliary beat frequency of human middle-ear mucosa measured in vitro. J Laryngol Otol 1984; 98: 853-856.

13 Rutland J, Griffin WM, Cole PJ. Human ciliary beat frequency in epithelium from intrathoracic and extrathoracic airways. Am Rev Respir Dis 1982; 125: 100-105.

14 Deitmer T. A method for standardizing cytologic sampling for the estimation of nasal ciliary activity. Arch Otorhinolaryngol 1986; 243: 288-292.

15 Winters SL, Davis CW, Boucher RC. Mechanosensitivity of mouse tracheal ciliary beat frequency: roles for $\mathrm{Ca}^{2+}$, purinergic signaling, tonicity, and viscosity. Am J Physiol Lung Cell Mol Physiol 2007; 292: L614-L624. 